ENCYCIOPEDDE Encyclopédie berbère

BERBERE

$25 \mid 2003$

25 | Iseqqemâren - Juba

\title{
Jeux chez les Touaregs
}

E. Bernus

\section{(2) OpenEdition}

Journals

Édition électronique

URL : http://journals.openedition.org/encyclopedieberbere/1514

DOI : 10.4000/encyclopedieberbere.1514

ISSN : 2262-7197

\section{Éditeur}

Peeters Publishers

\section{Édition imprimée}

Date de publication : 1 septembre 2003

Pagination : 3895-3903

ISBN : 2-7449-0424-4

ISSN : 1015-7344

\section{Référence électronique}

E. Bernus, « Jeux chez les Touaregs », Encyclopédie berbère [En ligne], 25 | 2003, document J10d, mis en ligne le 01 juin 2011, consulté le 24 septembre 2020. URL : http://journals.openedition.org/ encyclopedieberbere/1514; DOI : https://doi.org/10.4000/encyclopedieberbere.1514

Ce document a été généré automatiquement le 24 septembre 2020

(c) Tous droits réservés 


\section{Jeux chez les Touaregs}

\section{E. Bernus}

1 Parmi les jeux pratiqués par les Touaregs, il faut distinguer les jeux sportifs qui opposent deux équipes ou deux hommes, des jeux de société dans un cadre inscrit dans le sable, et les jeux d'esprit, véritables joutes verbales; enfin, il faut signaler la construction par les enfants de jouets. Un certain nombre de jeux sont connus chez d'autres populations africaines. L'ouvrage de Charles Béart, Jeux et jouets de l'Ouest africain (1955), nous permet d'utiles comparaisons. Il est donc intéressant de noter comment ces jeux, chez les Touaregs, possèdent une originalité dans la forme du jeu et dans le vocabulaire qui montre que ces jeux représentent une projection dans un univers de pasteurs. Par exemple, dans tous les jeux, on dit pour signaler le vainqueur, išwa, il a bu : la victoire, c'est boire, la défaite, c'est d'être privé d'eau' ${ }^{1}$.

\section{Les jeux sportifs}

2 karey a pu être comparé au hockey.

«Deux camps (aman); le nombre des joueurs varie de 20 à 25 pour chaque camp; les passes se disputent sur toute la longueur du terrain délimité par la ligne des tentes du campement, par exemple, et une ligne d'autres tentes ou une limite naturelle marquée par de la végétation par exemple. La balle est faite d'un morceau de peau de bovin, roulé et lié ; les sticks, tourning, charges sont autorisés et il y a souvent des blessés. Ce jeu n'est pratiqué que par les Imâjer'en et les Arabes Deremchaka et Yaéddès, en hivernage surtout quand on se rencontre sur les terres salées au cours de la saison heureuse » (F. Nicolas, 1950 : 183-184).

On remarque que les camps de ce jeu sont appelés aman, «l'eau », de telle sorte que la vainqueur a bu (išwa). La crosse est faite d'un bâton noueux, souvent pris dans une racine. Karey aujourd'hui n'est plus pratiqué sous cette forme organisée.

On voit cependant des jeunes gens qui jouent, comme à l'entraînement, les uns contre les autres avec un caillou ou un fruit du palmier doum (Hyphaene thebaica) et des crosses. 
5 La lutte (photo $\mathrm{n}^{\circ} 1$ ) est connue chez tous les Touaregs. Elle est appelée tabellant chez les Iwellemmeden (F. Nicolas, 1950: 184) et timsâl dans l'Ahaggar (Foucauld \& Calassanti-Motylinski, $1984: 80$ ).

«Les enfants comme les hommes pratiquent la lutte. L'un d'eux pose la main sur les épaules de son camarade. Tantôt, il le saisit par les épaules, tantôt par le cou. L'autre pose la main sur l'aisselle de son adversaire. Chacun tente de renverser son compagnon. Chacun, à tour de rôle, a le droit de poser en premier la main sur le coude l'autre. [...]. C'est celui qui a renversé son adversaire qui a gagné » (Foucauld, Calassanti-Motylinski, 1984 : 80).

6 Il existait autrefois des jeux d'escrime pratiqués avec épée et bouclier (Nicolas, 1950 : 184 [fig. 50]).

\section{Jeux de société avec inscription dans le sable}

7 Ces jeux sont très nombreux et il est impossible de tous les décrire. Certains sont connus chez d'autres populations mais ne se jouent pas sur le sol comme chez les Touaregs. Nous privilégierions deux jeux parmi les plus connus, Tihulelin ti $n$ äzgag et dära.

8 Tihulelin ti n äzgag appartient à un type de jeu connu dans de nombreuses populations sous le nom de mankala. "Il appartient au sous-type appelé dans la littérature africaniste wari (ou wali, wuri, wuré, awélé) »(Casajus, 1988: 23). Awélé est souvent cité, car chez les Akan, il a donné lieu à la fabrication de très beaux objets sculptés en bois avec les douze cases destinées à recevoir les pions. Le jeu touareg est pratiqué par les enfants et surtout par les fillettes : il comprend huit cases, plus rarement douze, creusées dans le sable: les pions sont le plus souvent constitués de crottes de chameaux.

"Chaque joueur dispose de 24 pions (36 pour les jeux à 12 cases) et les dépose dans les 4 cases situées devant lui à raison de 6 par case. Durant la partie, il les fait progresser dans un sens - direct ou rétrograde - qui reste le même pour lui et qui est le sens opposé à celui alloué à son adversaire. [...] La partie se poursuit, chaque joueur vidant une des quatre cases situées devant lui et déposant les pions, un par un, dans les cases suivantes. De la case où l'on dépose le dernier des pions prélevés, on dit qu'"on y fait étape", en utilisant le verbe $a d w u$, qui signifie ordinairement “arriver à l'étape du soir, rentrer chez soi le soir". [...] Le joueur doit chercher à obtenir, dans la case où il a fait étape, un nombre de pions égal à 2,4 ou 6 (en comptant le pion déposé). Il retire alors ces 2,4 ou 6 pions dans l'avant-dernière case atteinte, il les retire également du jeu. Il fait de même dans l'antépénultième si 2, 4 ou 6 s'y trouvent également, et ainsi de suite, en remontant. A l'issue de certains coups heureux, le joueur peut vider toutes les cases. On dit d'un joueur marquant ainsi des points qu'il "a mangé les pions" (icca), ou bien qu'il "a bu" (isha)

»(Casajus $1988: 26-27)$.

Ce jeu et connu dans toute l'Afrique, mais ici il se joue dans le sable et est réservé aux enfants.

Dära est un jeu connu de nombreuses populations africaines : soudanaises, sahéliennes ou sahariennes. Il est appelé dära chez les Touaregs méridionaux (Bernus, 1975 : 167-176) et karad, chez les Kel Ahaggar, c'est-à-dire "trois", en référence à l'alignement de trois pions nécessaires à l'enlèvement d'un pion adverse (Foucauld, 1951-1952 : II, 868). Dans le cas le plus courant, les joueurs inscrivent trente trous dans le sol $(6 \times 5)$ et chaque joueur dispose de douze pions. 
11 Signalons toutefois que Foucauld décrit ce jeu chez les Kel Ahaggar sur un casier de 42 cases avec 36 pions, dont 18 d'une couleur et 18 d'une autre et Nicolas (1950:182) cite le dära chez le Iwellemmeden Kel Denneg comme un jeu de 12 cases avec variantes : 42 trous et 18 pions. Les femmes établissent leur cadre avec 8 cases seulement.

12 La partie se déroule en deux phases. D'abord, chaque joueur place, un à un, alternativement, ses pions, sans en aligner trois. Ensuite, chacun déplace ses pions pour essayer de réaliser une série de trois, ce qui donne le droit d'enlever un pion à l'adversaire. Un bon placement de ses pions dans la première phase peut donner un avantage décisif.

13 Dans l'Ahaggar, chaque case est appelée éhen, la tente, alors que chez les Iwellemmeden elle reçoit le nom d'anu, le puits. Les pions sont le plus souvent formés de crottes de chameaux (ayereg, plur. iүeregan), de bâtonnets ou de cailloux.

14 Les joueurs sont de jeunes gens (photo $n^{\circ} 2$ ), quelquefois des hommes d'âge mûr, entourés de spectateurs qui commentent la partie et conseillent les joueurs. Les femmes sont absentes de ces groupes de joueurs qui ont installé leur damier près des tentes.

«La première phase du jeu, la mise en place des pions, se déroule rapidement chaque joueur lançant alternativement avec vigueur son pion dans la case choisie ; parfois, cependant, un joueur garde la main en l'air, suspendue au-dessus du damier, avant de porter son choix sur une case : c'est un geste arrêté, en suspens, avant la décision finale.

La deuxième phase, si la première n'a pas déjà mis en place le vainqueur, s'effectue plus lentement, avec circonspection. Les joueurs, à tour de rôle, prennent un pion et tapotent la case vide, le temps de la réflexion. [...]. Le premier joueur qui a gagné 4 parties est vainqueur d'une grande partie que l'on comptabilise comme une victoire. La revanche suit immédiatement et, en général, on fixe à l'avance le nombre de grandes parties à atteindre pour être définitivement vainqueur » (Bernus, 1975 : 170).

15 C'est dans le vocabulaire qu'apparaît l'inscription de ce jeu dans le contexte d'une civilisation pastorale.

« Dans la première phase de la partie, il est interdit de constituer des séries de trois. : si un joueur, par mégarde, aligne trois pions, son adversaire lui dit: "tiggezegh éhan $n$ tadaggalt-nek", "tu es entré dans la tente de ta belle-mère". Par là, il signifie à son adversaire qu'il fait une chose interdite, puisque pénétrer dans la tente de sa bellemère est un acte qui va à l'encontre de tous les usages de la bienséance.

17 Chaque partie est nommée alem (plur. imnas), chameau. Du joueur qui marque le premier, on dit : ila alem, il possède un chameau. En cas d'égalité, 1 à 1 , on dira alem $d$ alem. Une grande partie, gagnée par le premier qui atteint 4 points, est dite tadrert ou okkoz imnas, quatre chameaux.

18 La case est dite $a n u$, le puits. Lorsqu'un joueur prend un pion à l'adversaire après avoir aligné trois des siens, on dit: "ishwa", "il a bu". En revanche, si au cours d'une partie primaire, (alem), un joueur un joueur n'a pu se saisir d'aucun pion adverse, on dit : “wer ishwa”, “il n'a pas bu”, ou aghraf, c'est-à-dire, en parlant d'un animal, il ne boit pas quotidiennement ${ }^{2}$. [...].

19 Lorsqu'un joueur n'a pu empêcher son adversaire d'aligner trois de ses pions, il lui dit : “eshishweq-q aman”, “je t'ai fait boire l'eau”, à moins que ce dernier ait pris les devants : “ikf-i aman”, “il m'a donné l'eau". 
1. Lutte entre deux jeunes garçons des Tahabanat. In Athes, Niger. Photo E. Bernus.

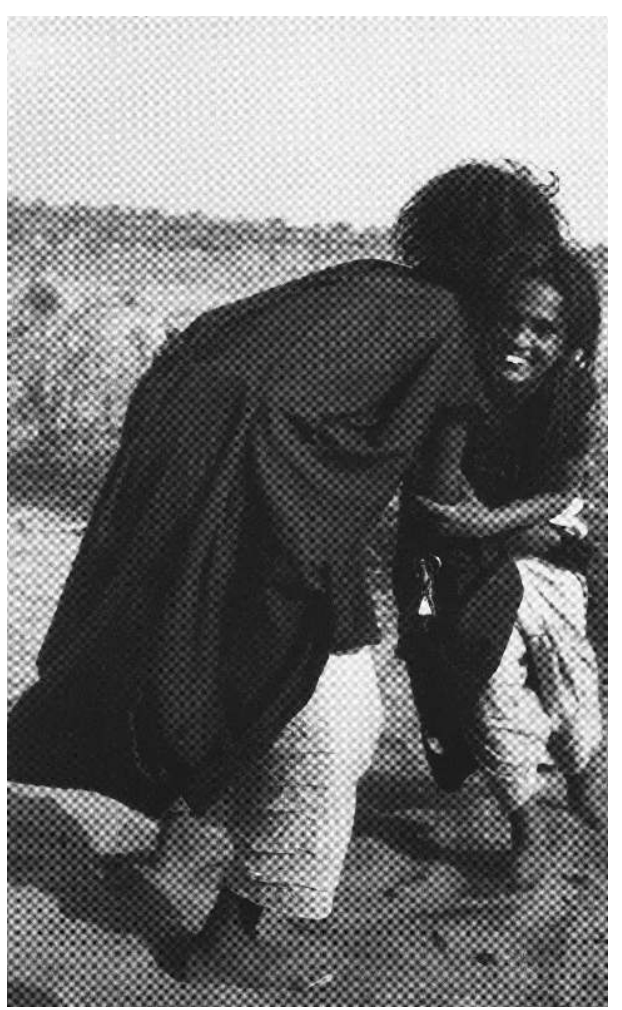

2. Partie de dära chez les Touaregs de l'Azawagh. Sud d'In Gall, Niger. Photo E. Bernus.

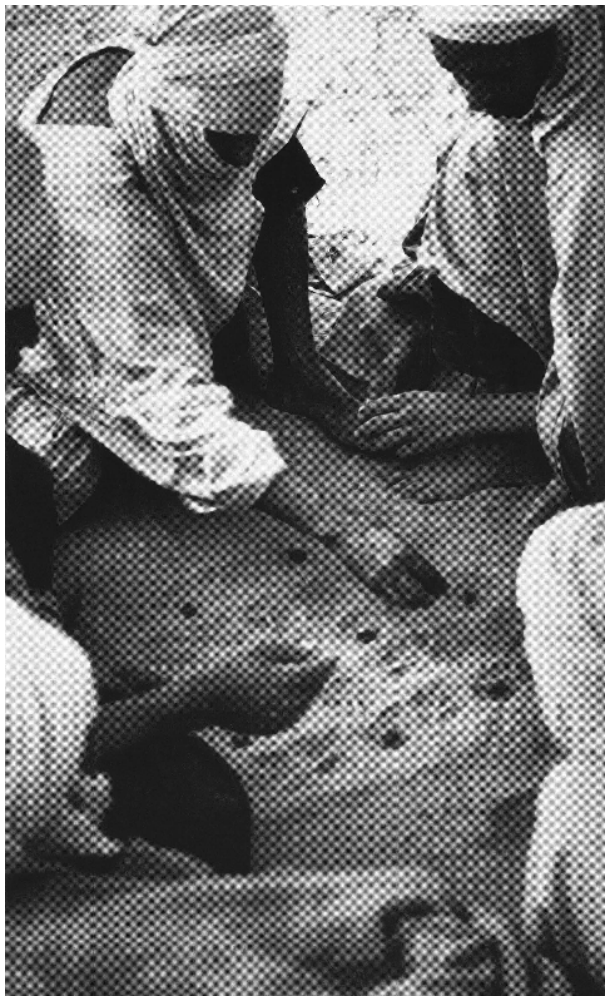

20 La position qui permet à un joueur de gagner est souvent constituée par un pion mobile, qui dans chaque mouvement va compléter une série de deux pions. C'est-à-dire 
qu'à chaque déplacement il réalise une série de trois et permet de prendre un pion à l'adversaire. Le pion qui navigue d'une case à l'autre est dit tebagawt, la jument, et le mouvement alternatif est appelé asri tebagawt, fait galoper la jument.

L'adversaire cherche alors à stopper ce mouvement et à placer un pion dans la case vide qui permet ce va-et-vient. On lui dit: "eghtes tebagawt", "coupe la jument" : eghtes signifie couper avec un couteau, un sabre. À l'occasion d'un mariage, le jeune époux, amène un ou plusieurs animaux dans le campement de la jeune mariée : lâchés on les poursuit et on leur coupe le jarret d'un coup de sabre avant de les immoler. Un tel animal est appelé taghtest.

Lorsque aucune parade n'a été possible, et que la jument permet de saisir un pion à chaque coup, on dit : imeksha, il a été mangé » (ibidem : 172-173).

D'autres jeux ont été décrits par Casajus (1988:23-49) et nous nous contenterons de les signaler. Les Arrawan, l'oljinnat, une forme ludique de la divination, le joueur imprimant la forme de sa main droite sur le sol ; les Iši baguettes en guise de dés, où ici aussi des combinaisons se rapportent à des animaux domestiques (vache, cheval blanc, chevrette, âne, cheval noir, chameau) ; le Fagošya, qui utilise aussi des baguettes dans un autre jeu de divination ; Takadänt, qui se joue à deux équipes de deux avec des cases en ligne appelées amajey (noble) et ékli (esclave); l' Amyar, où on joue seul comme dans nos jeux de " patiences "; enfin, sept petits jeux de veillée : Éhän en-gän, Koroma-gulbi, Tabatult, Forforam, Tir-degis, Asawad n emel, Bâssaү-tät. Igugelan, les jumeaux, met en présence un joueur qui doit trouver ce que représente un cercle que lui désigne un meneur de jeu alors qu'il a le dos tourné : les animaux concernés appartiennent aux quatre principaux types d'animaux sahéliens : chameaux, vaches, moutons, chèvres (Bernus, $1983: 15-20$ ).

\section{Jeux d'esprit}

Parmi les jeux d'esprit, il faut signaler les devinettes que le soir, à la veillée, les jeunes gens ont l'habitude se lancer. Elles sont appelées dans l'Ahaggar tunz̧art (plur. tunz arin), tanz̧urt (plur. tinz̧oren) chez les Touaregs maliens ou encore tamekerra (plur. timekarrawen) ou imeslan, chez les Iwellemmeden Kel Denneg.

Les devinettes constituent un dialogue: lorsqu'on pose une devinette, on commence par un appel, puis on énonce la devinette et enfin, on demande la réponse. L'appel varie d'une région à l'autre :

meslan, meslan : devinez, devinez (chez les Iwellemmeden Kel Denneg)

enzurey kay : je pose une devinette à toi (chez les Touaregs maliens).

La question, elle, est invariable : Ma imôs ? Qu'est-ce que c'est?

27 Les devinettes désignent des phénomènes naturels :

meslan, meslan / en tebutut $n$ amaḍal / Ma imôs ? / täyțțuft.

Devinez, devinez / le nombril de la terre, qu'est-ce que c'est? / La fourmilière.

En zone sahélo-saharienne, les fourmilières se présentent comme un monticule de forme circulaire avec au centre, un orifice par lequel pénètre les insectes.

Autre devinette connue :

meslan, meslan / $n$ zägretnin / télé daw-s / Ma imôs ? / anu 
Devinez, devinez / quelque chose de profond / l'ombre sous lui / qu'est-ce que c'est? / Le puits.

3. Chameau fabriqué avec une crotte de chameau et des épines d'aboray (Balanites aegyptiaca). Photo E. Bernus.

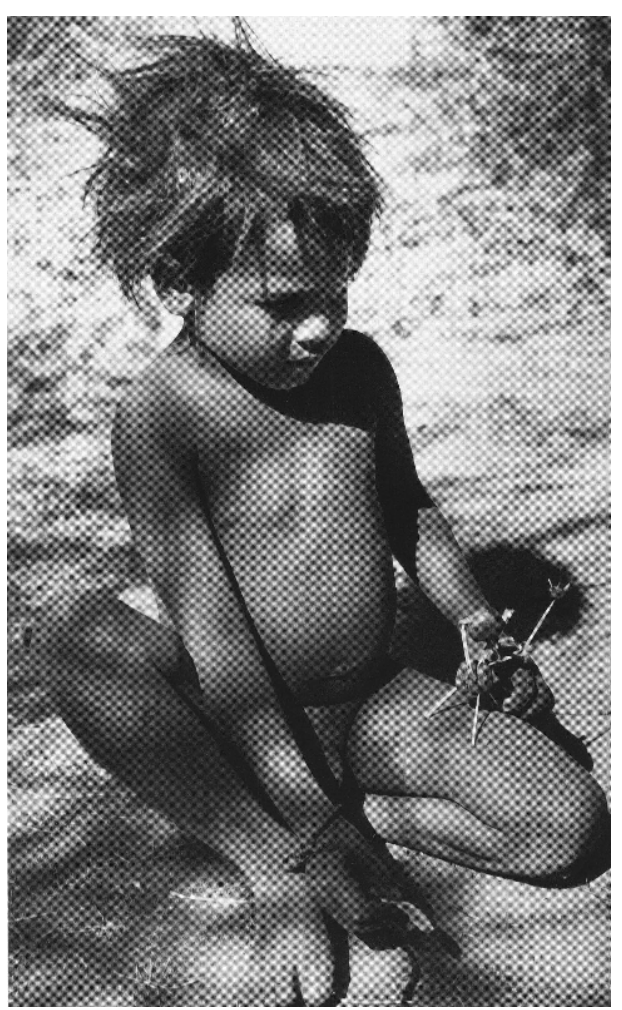


4. Modèle réduit de tente chez les Touaregs de l'Azawagh. Photo E. Bernus.

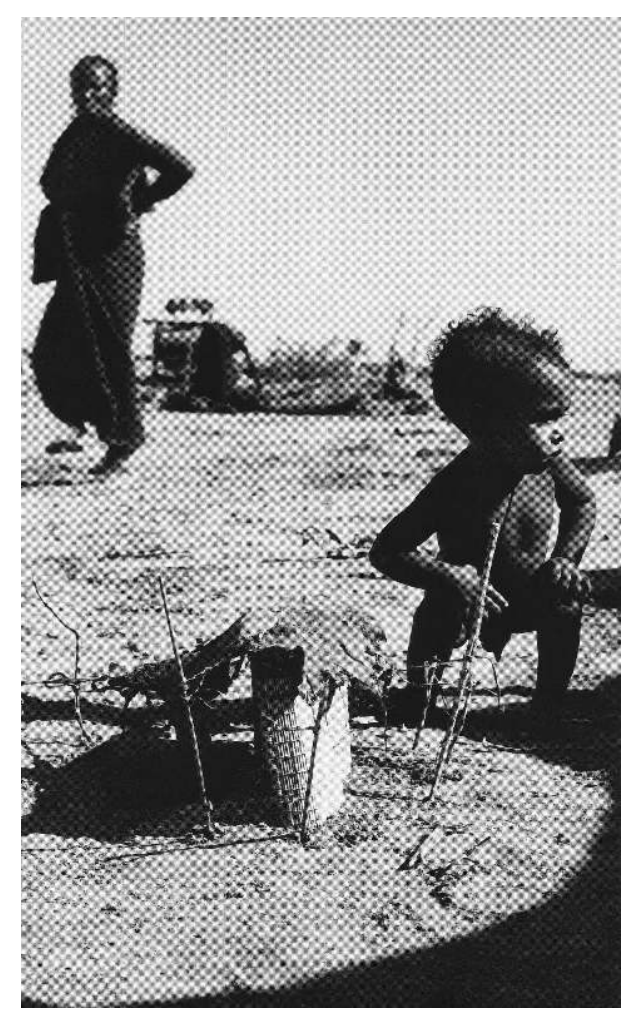

ou encore :

meslan, meslan / $\mathrm{n}$ ahug imdayen / Ma imôs ? / alked.

Devinez, devinez / le poulain qui est attaché / qu'est-ce que c'est ? / la courge sauvage. La courge sauvage (alked, Citrullus lanatus), comme la coloquinte (tagellet, Citrullus colocynthis) repose sur le sol avec une longue tige comme la longe d'un cheval.

\section{Jouets (voir notice J. 12 « Jouets »)}

31 Les jouets construits par les enfants son très nombreux : poupées fabriquées avec des chiffons, animaux domestiques façonnés dans l'argile ou chameau fait d'une crotte de chameau et d'épines d'aboray (Balanites aegyptiaca) (photo $\mathrm{n}^{\circ} 3$ ). On observe également de construction de modèles-réduits de tente avec, sous le vélum, le lit et la natteparavent (asabar) (photo $\mathrm{n}^{\circ} 4$ ). La liste est bien sûr incomplète.

\section{Conclusion}

Les jeux sont le miroir d'une société. Leur inscription dans le sable, la nature des pions sont le témoignage du milieu aride et des matériaux disponibles (crottes de chameaux, bâtonnets, cailloux). La référence constante aux animaux domestiques, à l'eau, au puits, à l'abreuvement montre l'intérêt manifesté par une société pastorale aux troupeaux dont dépend son existence. Enfin, la richesse des jeux d'esprit, joutes oratoires entre jeunes gens, dont nous avons donné qu'un exemple, révèle les Kel awal, les «Gens de la parole » que sont les Touaregs. 


\section{BIBLIOGRAPHIE}

BEART Ch., 1955, Jeux et jouets de l'Ouest africain, Dakar, Mémoire de l'IFAN nº 42, 2 tomes.

BELLIN P., 1963, «L'enfant touareg à travers ses jeux », in Journal de la Société des Africanistes, Paris, tome XXXIII, fasc. $1: 47-104$.

BERNUS E., 1989, « Paroles convenues. Mots et jeux de mots touaregs », in Graines de parole.

Puissance du verbe et tradition orale, Ecrits pour Geneviève Calame-Griaule, Paris, éditions du CNRS :

79-89.

BERNUS E., 1983, « Jeu et élevage. Igugelan, jeu touareg » in Bulletin des Études Africaines, vol. III, n 5, INALCO, Paris : 15-20.

BERNUS E., 1975, «Jeu et élevage. Vocabulaire d'élevage utilisé dans un jeu de quadrillage par les Touaregs ", in Journal d'Agriculture tropicale et de Botanique appliquée, Paris, Muséum National d'Histoire Naturelle, vol. XXII, nº 4-5-6 : 167-176.

CASAJUS D., 1988, « Jeux touaregs de la région d'Agadez » in Journal des Africanistes, Paris, tome LVIII, fasc. $1: 23-49$.

FOUCAULD CH. DE \& CALASSANTI-MOTYLINSKI A. de, 1984, Textes touaregs en prose, édition critique, traduction par S. Chaker, H. Claudot M. Gast, Aix-en-Provence, Édisud : 359 p.

FOUCAULD Ch. de, 1951-52, Dictionnaire touareg-français. Dialecte de l'Ahaggar, Paris, Imprimerie Nationale, 4 vol. : 2024 p.

NICOLAS F., 1950, Tamesna, Les Ioullemmeden de l'Est ou Touâreg « Kel Dinnik », Pris, Imprimerie Nationale, photos, figures : $279 \mathrm{p}$.

NICOLAS F., 1956, « Textes ethnographiques de la tamajeq des Ioullemmeden de l'Est », in Anthropos $\mathrm{n}^{\circ}$ 51, LIX ; « Jeu » : 949-950.

\section{NOTES}

1. Cet usage du verbe sew, "boire » est commun à tous les Berbères (cf. "Jeux en Kabylie »), NDLR.

2. Le nom verbal, désignant cet abreuvement non quotidien est connu en terme d'élevage, taghreft, qui s'oppose à timeshwit, abreuvement quotidien.

\section{INDEX}

Mots-clés : Algérie, Ayt Mgild, Enfant, Ethnographie, Kabylie, Moyen-Atlas, Sahara, Touareg 